IJMMS 25:5 (2001) 323-329

PII. S0161171201003350

http://ijmms.hindawi.com

(c) Hindawi Publishing Corp.

\title{
ON THE STRUCTURE OF THE TOTALLY ORDERED SET OF UNIMODAL CYCLES
}

\author{
IRENE MULVEY
}

(Received 26 April 1999)

\begin{abstract}
We continue the study of a class of unimodal cycles where each cycle in the class is forced by every unimodal cycle not in the class. For every order, we identify the cycle in the class of that order, which is maximal with respect to the forcing relation.
\end{abstract}

2000 Mathematics Subject Classification. Primary 37E05, 37E15.

1. Introduction. In 1964, Šarkovs'kil defined a linear order on the set of natural numbers:

$$
3 \prec 5 \prec 7 \prec \cdots \prec 2 \cdot 3 \prec 2 \cdot 5 \cdots \prec \cdots \prec 2^{2} \cdot 3 \prec 2^{2} \cdot 5 \cdots \prec \cdots \cdots \prec 2^{3} \prec 2^{2} \prec 2 \prec 1
$$

and proved the following theorem.

THEOREM 1.1 (Šarkovs'kil [5]). Let $f: \mathbb{R} \rightarrow \mathbb{R}$ be a continuous map. The set of (least) periods of $f$ is a tail of the above order. Conversely, for every tail of the above order, there is a continuous map $f: \mathbb{R} \rightarrow \mathbb{R}$ having exactly those periods.

In 1987, Baldwin [2] considered not only the least period of a periodic point, but also the orbit type. He defined the forcing relation on finite cyclic permutations (cycles), proved that the forcing relation is a partial order, and provided an algorithm to decide when one cycle forces another.

A cycle is unimodal if the canonical linear map it determines has exactly one turning point. Throughout this paper, we assume a unimodal cycle has exactly one turning point and it is a maximum. It is shown in [3] that the forcing relation is a total order on the set of unimodal cycles.

In [4], we described a class of unimodal cycles, where every cycle in the class is forced by every unimodal cycle not in the class. In this paper, for each order $n$, we identify the maximal cycle of order $n$ in this class with respect to the forcing relation.

2. Definitions. Let $f: I \rightarrow I$ be a continuous map of a compact interval to itself. We define $f^{0}(x)=x$ and for $n \in \mathbb{N}, n \geq 1, f^{n}(x)=f\left(f^{n-1}(x)\right)$. If there exists $k \in \mathbb{N}$ such that $f^{k}(x)=x$, then we say $x$ is periodic for $f$ and $x$ has least period $s$, where $s$ is smallest element of $\mathbb{N}$ such that $f^{s}(x)=x$. For $x \in I$, the orbit of $x$ is the set $\left\{f^{n}(x) \mid n \geq 0\right\}$. If $x$ is periodic with least period $s$, then the orbit of $x$ is the finite set $X=\left\{x, f(x), f^{2}(x), \ldots, f^{s-1}(x)\right\}$.

A cycle of order $m$ is a bijection $\eta:\{1,2, \ldots, m\} \rightarrow\{1,2, \ldots, m\}$ such that $\eta^{k}(1) \neq 1$ for $1 \leq k<m$. We denote a cycle $\eta$ by $\eta=\left(k_{1}, k_{2}, \ldots, k_{m}\right)$, where $\eta\left(k_{i}\right)=k_{i+1}$ and 
$\eta\left(k_{m}\right)=k_{1}$. We assume, without loss of generality, that $k_{1}=1$. Write the elements of a periodic orbit $X$ in increasing order: $x_{1}<x_{2}<\cdots<x_{s}$. We say $X$ has orbit type $\eta$ if $\eta$ is a cycle of order $s$ and for each $i \in\{1,2, \ldots, s\}, f\left(x_{i}\right)=x_{\eta(i)}$. In fact, we say each $x_{i} \in X$ has orbit type $\eta$.

The forcing relation on cycles is defined as follows: $\theta$ forces $\eta$ if and only if every continuous map of the interval that has a periodic orbit of type $\theta$ has a periodic orbit of type $\eta$. Baldwin [2] proved that this relation induces a partial order on the set of cycles.

Let $\theta$ be a cycle of order $n$. The canonical $\theta$-linear map $L_{\theta}$ is defined by $L_{\theta}:[1, n] \rightarrow$ $[1, n]$, where $L_{\theta}=\theta$ on $\{1,2, \ldots, n\}$ and $L_{\theta}$ is linear on $[i, i+1]$ for $1 \leq i \leq n-1$. It is known that $\theta$ forces $\eta$ if and only if $L_{\theta}$ has a periodic orbit of orbit type $\eta$ [1]. A cycle $\theta$ is called unimodal if $L_{\theta}$ has exactly one turning point. Throughout this paper, every unimodal cycle is assumed to have one turning point and it is a maximum. The forcing relation defined by Baldwin induces a total order on the set of unimodal cycles [3].

Let $\theta$ be a cycle of order $n$.

DefinItion 2.1. The $R L$-pattern for $\theta$ is the element $S=S_{1} S_{2} \cdots S_{n} \in\{R, L\}^{n}$ satisfying

$$
S_{i}= \begin{cases}R & \text { if } \theta^{i}(1)>\theta^{i-1}(1), \\ L & \text { if } \theta^{i}(1)<\theta^{i-1}(1) .\end{cases}
$$

For example, the $R L$-pattern for $\theta=(12354)$ is $R R R L L$. Both $\theta=(15234)$ and $\theta=$ (13245) have the $R L$-pattern $R L R R L$. Every $R L$-pattern begins with $R$ and ends with $L$. If $X$ is a periodic orbit of orbit type $\theta$, then the $R L$-pattern for $X$ is the $R L$-pattern for $\theta$.

3. Preliminaries. The next four results are proved in [4].

LEMMA 3.1. If $\theta$ is unimodal, then $L_{\theta}$ has a unique fixed point.

LEMMA 3.2. If $\theta$ is unimodal, then the RL-pattern for $\theta$ cannot contain two consecutive $L$ 's.

DeFinition 3.3. Let $C$ denote the class of unimodal cycles whose $R L$-pattern does not contain two consecutive $R$ 's.

LEMMA 3.4. Let $\theta \in C$ be of order $n$. Then

(1) $n$ is even and $n \geq 4$.

(2) $\theta(n-i)=i+1$ for $i=0,1,2, \ldots, k-1$.

(3) $\theta(1)=k+1$.

THEOREM 3.5. Let $\eta \in C$ and let $\theta$ be a unimodal cycle not in $C$. Then $\theta$ forces $\eta$.

DEFINITION 3.6. For even $n \geq 4$,

$$
\bar{\theta}_{n}=(1, k+1, k, k+2, k-1, k+3, k-2, \ldots, n-1,2, n) .
$$

REMARK 3.7. In the main theorem, we prove that $\bar{\theta}_{n}$ is the maximal cycle of order $n$ in $C$ with respect to the forcing relation. 
LEMMA 3.8. Let $\theta \in C$ be of order $n$. Then $\theta=\bar{\theta}_{n}$ if and only if $\theta(2)=n$.

Proof. The map $L_{\theta}$ is a bijection which maps $\{3,4, \ldots, k\}$ to $\{k+2, k+3, \ldots, n-1\}$. Since $\theta(2)=n$ and $\theta$ is unimodal, $L_{\theta}$ is decreasing on $\{3,4, \ldots, k\}$.

The next lemma restates Lemma 3.8 in the way we will often use it.

LEMmA 3.9. Let $\theta \in C$ be of order $n$. Then $\theta \neq \bar{\theta}_{n}$ if and only if there exists $i \in$ $\{3,4, \ldots, k\}$ such that $\theta(i)=n$.

\section{Main theorem}

LEMMA 4.1. Let $\theta \in C$ be of order $n$. Then the only periodic point for $L_{\theta}$ in $(k, k+1)$ is the unique fixed point for $L_{\theta}$.

Proof. $L_{\theta}(k+1)=k$ and $L_{\theta}(k) \geq k+2$. If $x<p$, then for $j \geq 0, L_{\theta}^{2 j}(x)<p$ and $L_{\theta}^{2 j+1}(x)>p$. Let $\bar{k}$ be the unique preimage of $k+1$ in $(k, p)$. If $x \in(k, \bar{k}]$, then $L_{\theta}(x) \geq k+1$ and $x$ is not periodic since $[1, k] \cup[k+1, n]$ is invariant under $L_{\theta}$. If $x \in(\bar{k}, p)$, then $L_{\theta}^{2}(x)<p$. If $L_{\theta}^{2}(x)<\bar{k}$, then $x$ is not periodic. If $L_{\theta}^{2}(x) \in(\bar{k}, p)$, then $\left(p-L_{\theta}^{2}(x)\right) \geq 4(p-x)$ since $L_{\theta}^{\prime} \leq-2$ on $(k, k+1)$.

REMARK 4.2. In the next few results, we assume $\theta \in C$ is of order $n, \theta \neq \bar{\theta}_{n}$, and $\theta$ forces $\bar{\theta}_{n}$. By Definition 3.6 this means that $L_{\theta}$ has a periodic orbit $X$ of type $\bar{\theta}_{n}$. As standard notation, we write the elements of $X$ in increasing order and label them as follows:

$$
X=\left\{x_{1}<x_{2}<\cdots<x_{n}\right\}
$$

where

$$
L_{\theta}\left(x_{i}\right)=x_{\bar{\theta}_{n}(i)}
$$

LEMмA 4.3. Suppose that $\theta \in C$ is of order $n$ and $\theta \neq \bar{\theta}_{n}$. If $\theta$ forces $\bar{\theta}_{n}$, then $\left\{x_{1}, x_{2}, \ldots, x_{k}\right\} \subseteq(1, k)$ and $\left\{x_{k+1}, x_{k+2}, \ldots, x_{n}\right\} \subseteq(k+1, n)$.

PROoF. Since $X=\left\{x_{1}<x_{2}<\cdots<x_{n}\right\}$ is an orbit of type $\bar{\theta}_{n}$, we have

$$
\begin{array}{r}
\left\{x_{1}, x_{2}, \ldots, x_{k}\right\} \subseteq\left\{x \mid L_{\theta}(x)>x \text { and } L_{\theta}^{2}(x)<L_{\theta}(x)\right\} \subseteq[1, p], \\
\left\{x_{k+1}, x_{k+2}, \ldots, x_{n}\right\} \subseteq\left\{x \mid L_{\theta}(x)<x \text { and } L_{\theta}^{2}(x)>L_{\theta}(x)\right\} \subseteq[p, n] .
\end{array}
$$

But by Lemma 4.1 , no $x_{i}$ can be in $(k, k+1)$.

LEMMA 4.4. Let $\theta \in C$ be of order $n$ and $\theta \neq \bar{\theta}_{n}$. If $\theta$ forces $\bar{\theta}_{n}$, then there are at most two $x_{i}$ in $(1,3)$.

Proof. If not, then (at least) $x_{1}<x_{2}<x_{3}$ are in (1,3). By Lemma 3.9, $L_{\theta}$ is increasing on $(1,3)$. So $L_{\theta}\left(x_{3}\right)>L_{\theta}\left(x_{2}\right)$ or $x_{\bar{\theta}_{n}(3)}>x_{\bar{\theta}_{n}(2)}$ or $x_{n-1}>x_{n}$. Hence, there are at most two $x_{i}$ in $(1,3)$.

The next definition and lemma could have been proved just after Lemma 3.4, but we place them here since they were not needed until now. 
DeFinition 4.5. For $\theta \in C$ of order $n$, let $P_{j}=\{i \mid \theta(i) \geq j\}$.

LEMMA 4.6. Let $\theta \in C$ be of order $n$. Then

(1) the order of $P_{j}$ is $n-(j-1)$,

(2) for $1 \leq j \leq n$, either the largest element in $P_{j}$ or the smallest element in $P_{j}$ maps to $j$ under $L_{\theta}$.

Proof. The proof of (1) is obvious.

(2) is proved inductively. We know that $P_{1}=\{1,2, \ldots, n\}$ and $L_{\theta}(n)=1$, so (2) is true for $j=1$. For $j>1, P_{j}$ is a finite sequence of consecutive integers since each $P_{j}$ is derived from $P_{j-1}$ by removing the maximum or minimum of $P_{j-1}$ from $P_{j-1}$. Assume that (2) is true for $j$. Consider $P_{j+1}=\{i \mid \theta(i) \geq j+1\}$. Let $a=\min P_{j+1}$ and $b=\max P_{j+1}$. If neither $a$ nor $b$ maps to $j+1$ under $L_{\theta}$, then $L_{\theta}(a)>j+1$ and $L_{\theta}(b)>j+1$. But there exists an integer $c \in(a, b)$ such that $L_{\theta}(c)=j+1$, and this contradicts the fact that $\theta$ is unimodal.

LEMMA 4.7. Let $\theta \in C$ be of order $n$ and let $k=n / 2$. Let $\bar{j} \geq 0$ be the largest integer such that $2+\bar{j}<k-\bar{j}$. Then for all $j=0,1,2, \ldots, \bar{j}$,

$$
L_{\theta}((2+j, k-j)) \subseteq(k+2+j, n] .
$$

Proof. For $j=0$, consider, by Lemma 3.4,

$$
P_{k+2}=\{i \mid \theta(i) \geq k+2\}=\{2,3, \ldots, k\} .
$$

So if $x \in(2, k)$, then $L_{\theta}(x)>k+2$. That is,

$$
L_{\theta}((2, k)) \subseteq(k+2, n] .
$$

Note that $P_{k+3}$ properly contains $\{3,4, \ldots, k-1\}$ since by Lemma 4.6 exactly one of $\{2, k\}$ maps to $k+2$ under $L_{\theta}$.

Inductively, suppose that $j$ satisfies $0 \leq j<\bar{j}$ and

$$
L_{\theta}((2+j, k-j)) \subseteq(k+2+j, n] .
$$

Consider

$$
P_{k+2+(j+1)}=\{i \mid \theta(i) \geq k+2+(j+1)\} \supset\{2+(j+1), \ldots, k-(j+1)\}
$$

(a proper containment). So if $x \in(2+(j+1), k-(j+1))$, then

$$
L_{\theta}(x)>k+2+(j+1) .
$$

That is,

$$
L_{\theta}((2+(j+1), k-(j+1))) \subseteq(k+2+(j+1), n],
$$

as needed.

LEMmA 4.8. Let $\theta \in C$ be of order $n$, and let $k=n / 2$. Assume that $\theta \neq \bar{\theta}_{n}$ and $\theta$ forces $\bar{\theta}_{n}$. Let $\bar{j} \geq 0$ be the largest integer such that $2+\bar{j}<k-\bar{j}$. Then for all $j=0,1,2, \ldots, \bar{j}$, there exists at most one $x_{i}$ in $(k-j-1, k-j)$. 
Proof. We have $\theta \in C$ of order $n, \theta \neq \bar{\theta}_{n}$, and $\theta$ forces $\bar{\theta}_{n}$. By Lemma 4.3, $\left\{x_{1}, x_{2}, \ldots, x_{k}\right\} \subseteq(1, k)$. Let $\bar{j}$ be the largest integer such that $2+j<k-\bar{j}$. The proof is inductive. First, for $j=0$, suppose that there are two or more $x_{i} \in(k-1, k)$. Then (at least) $x_{k}, x_{k-1}$ are in $(k-1, k)$. It follows from Lemma 3.4 that

$$
x_{i+1}, x_{k+2} \in(k+1, k+2)
$$

since $x_{k+1}$ and $x_{k+2}$ are preimages of $x_{k}$ and $x_{k-1}$ and

$$
L_{\theta}^{-1}([k-1, k])=[k+1, k+2] .
$$

Now, we have $x_{k} \in(k-1, k)$ and

$$
\theta\left(x_{k}\right)=x_{k+2} \in(k+1, k+2) .
$$

But

$$
\theta\left(x_{k}\right) \in L_{\theta}(k-1, k) \subseteq L_{\theta}(2, k) \subseteq(k+2, n]
$$

by Lemma 4.7 , which is a contradiction. Therefore, there is at most one $x_{i} \in(k-1, k)$.

Next, suppose that $\tilde{j}$ satisfies $0 \leq \tilde{j}<\bar{j}$ and for all $j=0,1,2, \ldots, \tilde{j}$, there is at most one $x_{i}$ in $(k-j-1, k-j)$. Suppose that there are two or more $x_{i}$ in $J=(k-\tilde{j}-2$, $k-\tilde{j}-1)$. There are $\tilde{j}+2$ ways for this to occur, since each of the $\tilde{j}+1$ intervals

$$
(k-1, k),(k-2, k-1), \ldots,(k-\tilde{j}-1, k-\tilde{j})
$$

contains at most one $x_{i}$. As in the case when $j=0$, the preimages of any elements in $J=(k-\tilde{j}-2, k-\tilde{j}-1)$ must be in $K=(k+\tilde{j}+2, k+\tilde{j}+3)$.

CASE $1\left(x_{k}, x_{k-1} \in J\right)$. Then $x_{k+1}, x_{k+2} \in K$.

CASE $2\left(x_{k-1}, x_{k-2} \in J\right)$. Then $x_{k+2}, x_{k+3} \in K$. and so on, until

CASE $\tilde{j}+2\left(x_{k-\tilde{j}-1}, x_{k-\tilde{j}-2} \in J\right)$. Then $x_{k+\tilde{j}+2}, x_{k+\tilde{j}+3} \in K$.

In every case there exists $x_{r} \in J$ and $L_{\theta}\left(x_{r}\right) \in K$. Now,

$$
L_{\theta}\left(x_{r}\right) \in L_{\theta}(J)=L_{\theta}(k-\tilde{j}-2, k-\tilde{j}-1) \subseteq L_{\theta}(2+\tilde{j}+1, k-\tilde{j}-1) \subseteq(k+2+\tilde{j}+1, n],
$$

by Lemma 4.7. So $L_{\theta}\left(x_{r}\right)>k+\tilde{j}+3$. But

$$
L_{\theta}\left(x_{r}\right) \in K=(k+\tilde{j}+2, k+\tilde{j}+3),
$$

which is a contradiction. This proves the lemma. Note that $2+(\tilde{j}+1)<k-(\tilde{j}+1)$ by hypothesis, so $2+\tilde{j}+1 \leq k-\tilde{j}-2$, as needed in (4.16).

LEMMA 4.9. Let $\theta \in C$ be of order $n$, and let $k=n / 2$. Assume that $\theta \neq \bar{\theta}_{n}$ and $\theta$ forces $\bar{\theta}_{n}$. Let $\bar{j}$ be the largest integer such that $2+\bar{j}<k-\bar{j}$. If $\theta(i)=n$, then $3 \leq i \leq k-\bar{j}-2$.

PRoof. There are at most two $x_{i}$ that are less than $i$, as in the proof of Lemma 4.4. So there are at least $(k-2) x_{i}$ in $(i, k)$. If $i>k-\bar{j}-2$, then there are at most $\bar{j}+$ 1 intervals and each interval contains at most one $x_{i}$. Since $k-2>\bar{j}+1$, this is a contradiction. 
LEMmA 4.10. Let $\theta \in C$ be of order $n$ and let $k=n / 2$. Assume that $\theta \neq \bar{\theta}_{n}$ and $\theta$ forces $\bar{\theta}_{n}$. Let $\bar{j}$ be the largest integer such that $2+\bar{j}<k-\bar{j}$. Then there is at most one $x_{i}$ in each of the intervals

$$
(3,4),(4,5), \ldots,(k-\bar{j}-2, k-\bar{j}-1) .
$$

Proof. Let $\bar{i}$ be such that $\theta(\bar{i})=n$. Then $x_{1}<\bar{i}<x_{3}$. By Lemma $4.9,3 \leq \bar{i} \leq k-\bar{j}-2$. Define $\tilde{i}$ by $\bar{i}=k-\bar{j}-\tilde{i}$. Suppose that there are at least two $x_{i} \in(\bar{i}, \bar{i}+1)$.

Either

$$
x_{2}, x_{3} \in(k-(\bar{j}+\tilde{i}), k-(\bar{j}+\tilde{i})+1),
$$

in which case

$$
x_{n-1}, x_{n-2} \in(k+(\bar{j}+\tilde{i}), k+(\bar{j}+\tilde{i})+1) \text {, }
$$

or

$$
x_{3}, x_{4} \in(k-(\bar{j}+\tilde{i}), k-(\bar{j}+\tilde{i})+1),
$$

in which case

$$
x_{n-2}, x_{n-3} \in(k+(\bar{j}+\tilde{i}), k+(\bar{j}+\tilde{i})+1) .
$$

In either case, there exists $x_{r} \in(k-(\bar{j}+\tilde{i}), k-(\bar{j}+\tilde{i})+1)$ such that

$$
\theta\left(x_{r}\right) \in(k+(\bar{j}+\tilde{i}), k+(\bar{j}+\tilde{i})+1) .
$$

This guarantees that

$$
\theta(k-(\bar{j}+\tilde{i})+1) \leq k+\bar{j}+\tilde{i}
$$

But, by (2) in Lemma 3.4,

$$
\theta(k+(\bar{j}+\tilde{i}))=\theta(n-k+(\bar{j}+\tilde{i}))=\theta(n-(k-\bar{j}-\tilde{i}))=k-\bar{j}-\tilde{i}+1=k-(\bar{j}+\tilde{i})+1 .
$$

Since $n \geq 4$,

$$
\theta(k-(\bar{j}+\tilde{i})+1)<k+\bar{j}+\tilde{i} .
$$

This leaves the set $A=\{2,3, \ldots, \bar{i}-1\}$ to be mapped bijectively by $\theta$ onto a set $B \supseteq\{k+\bar{j}+\tilde{i}, \ldots, n-1\}$. But

$$
\begin{gathered}
|A|=\bar{i}-2=k-\bar{j}-\tilde{i}-2, \\
|B| \geq(n-1)-(k+\bar{j}+\tilde{i})+1=(n-k)-\bar{j}-\tilde{i}=k-\bar{j}-\tilde{i} .
\end{gathered}
$$

Hence, there is at most one $x_{i} \in(\bar{i}, \bar{i}+1)$.

The proof continues in this fashion. We have proved that there is at most one $x_{i} \in(\bar{i}, \bar{i}+1)$. Suppose that there are at least two $x_{i} \in(\bar{i}+1, \bar{i}+2)$. There are three ways for this to occur. In each of these three cases, there exists $x_{r} \in(\bar{i}+1, \bar{i}+2)$ such that

$$
\theta\left(x_{r}\right) \in(k+\bar{j}+\tilde{i}-1, k+\bar{j}+\tilde{i})
$$

As in the previous case,

$$
\theta(\bar{i}+2) \leq k+\bar{j}+\tilde{i}-1 .
$$

This leaves the set $A=\{2,3, \ldots, \bar{i}-1\} \cup\{\bar{i}+1\}$ with $|A|=\bar{i}-1=k-\bar{j}-\tilde{i}-1$ to be mapped bijectively onto a set containing $B \supseteq\{k+\bar{j}+\tilde{i}-1, \ldots, n-1\}$, where $|B| \geq$ $k-\bar{j}+\tilde{i}$. 
THEOREM 4.11. Let $\theta \in C$ of order $n$. Then $\bar{\theta}_{n}$ forces $\theta$.

Proof. If $\theta=\bar{\theta}_{n}$, the conclusion holds trivially. Assume that $\theta \neq \bar{\theta}_{n}$ and suppose that $\bar{\theta}_{n}$ does not force $\theta$. Since forcing induces a total order on $C, \theta$ forces $\bar{\theta}_{n}$, so there exist $x_{1}<x_{2}<\cdots<x_{n}$ in $(1, n)$ such that for each $i, L_{\theta}\left(x_{i}\right)=x_{\bar{\theta}_{n}(i)}$. By Lemma 4.3, $\left\{x_{1}, x_{2}, \ldots, x_{k}\right\} \subseteq(1, k)$, where $k=n / 2$. By Lemma 4.4 , there are at most two $x_{i} \in(1,3)$. This leaves at least $k-2$ of the $x_{i}$ in the union of intervals $(3,4) \cup(4,5) \cup \cdots \cup(k-1, k)$. But each one of these $k-3$ intervals contains at most one $x_{i}$ by Lemmas 4.8 and 4.10.

\section{REFERENCES}

[1] L. Alsedà, J. Llibre, and M. Misiurewicz, Combinatorial Dynamics and Entropy in Dimension One, Advanced Series in Nonlinear Dynamics, vol. 5, World Scientific Publishing Co., Inc., River Edge, NJ, 1993. MR 95j:58042. Zbl 843.58034.

[2] S. Baldwin, Generalizations of a theorem of Sarkovskii on orbits of continuous real-valued functions, Discrete Math. 67 (1987), no. 2, 111-127. MR 89c:58057. Zbl 632.06005.

[3] P. Collet and J.-P. Eckmann, Iterated Maps on the Interval as Dynamical Systems, Progress in Physics, vol. 1, Birkhäuser, Boston, Mass., 1980. MR 82j:58078. Zbl 458.58002.

[4] I. Mulvey, Symbolic representation for a class of unimodal cycles, preprint, 1998.

[5] O. M. Šarkovs'kili, Co-existence of cycles of a continuous mapping of the line into itself, Ukrain. Mat. Z̆. 16 (1964), 61-71 (Russian). MR 28\#3121.

Irene Mulvey: Mathematics and Computer SCiences, College of ARTS AND SCiences, FAIRFIELD UNIVERSITY, NORTH BENSON ROAD, FAIRFIELD, CONNECTICUT 06430-5195, USA

E-mail address: mu1vey@fair1. fairfie1d.edu 


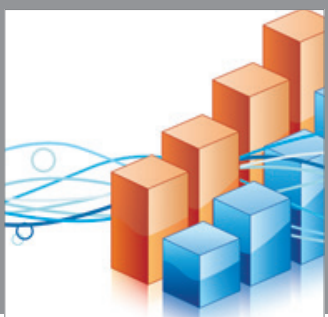

Advances in

Operations Research

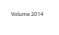

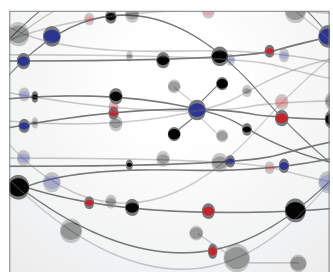

\section{The Scientific} World Journal
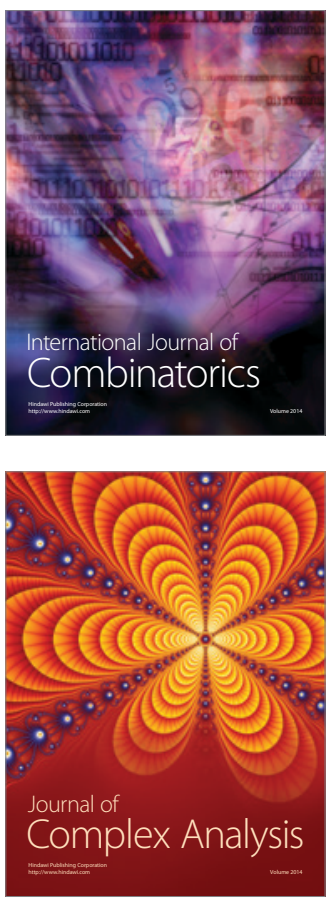

International Journal of

Mathematics and

Mathematical

Sciences
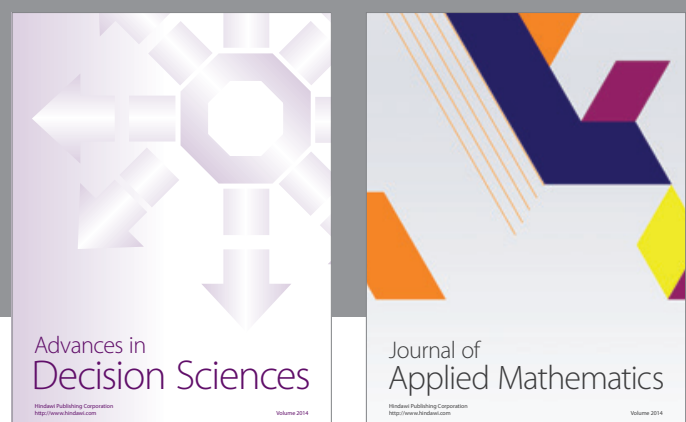

Journal of

Applied Mathematics
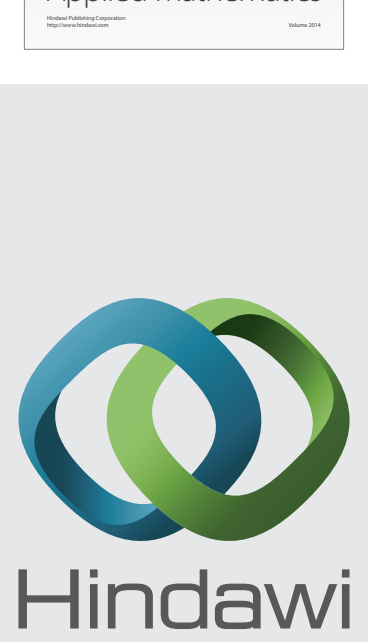

Submit your manuscripts at http://www.hindawi.com
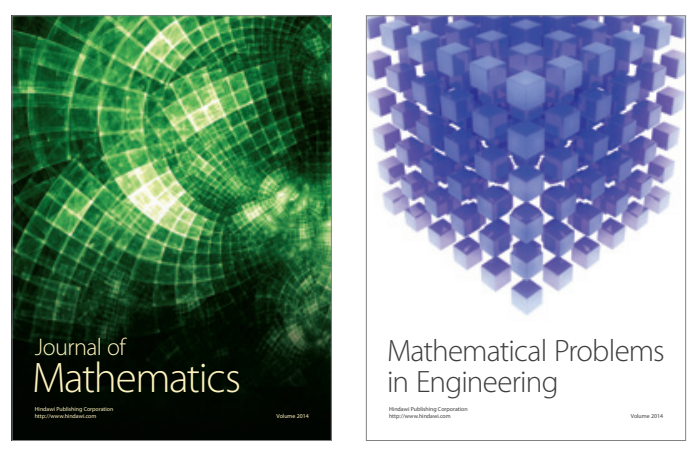

Mathematical Problems in Engineering
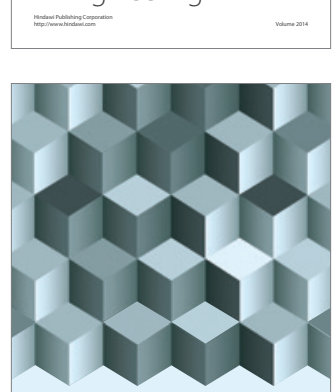

Journal of

Function Spaces
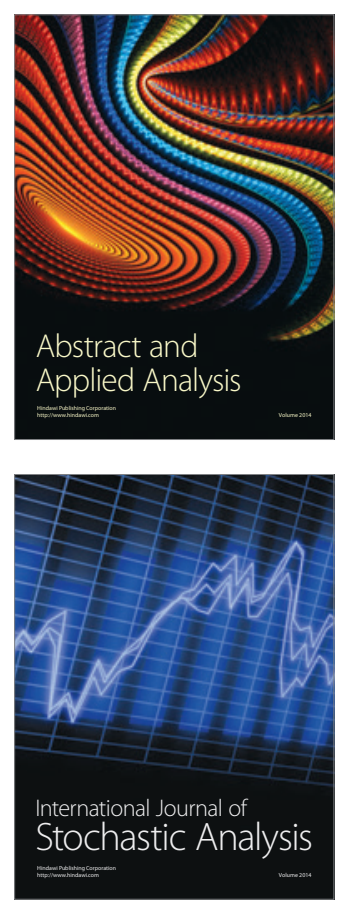

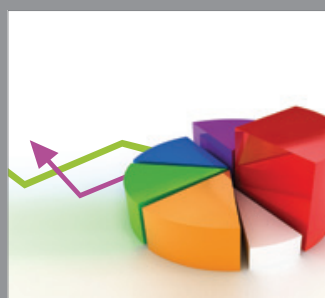

ournal of

Probability and Statistics

Promensencen
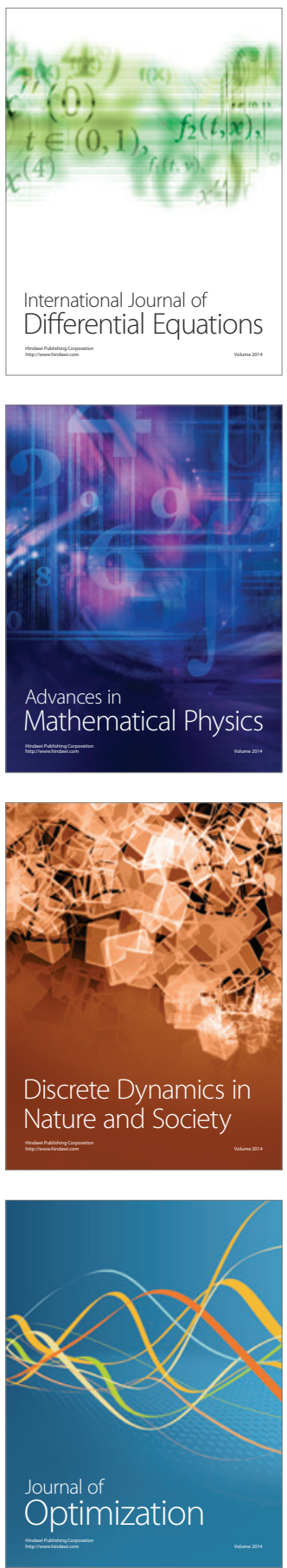